\title{
Humanités numériques
}

\section{Éditorial. Le troisième vient, c'est encore une première...}

Editorial. The Third Is upon Us, It's Yet Another First...

\section{(2) OpenEdition}

\section{Journals}

Édition électronique

URL : https://journals.openedition.org/revuehn/2120

DOI : 10.4000/revuehn.2120

ISSN : 2736-2337

Éditeur

Humanistica

Référence électronique

"Éditorial. Le troisième vient, c'est encore une première... », Humanités numériques [En ligne], 3 | 2021, mis en ligne le 01 mai 2021, consulté le 15 juillet 2021. URL : http://journals.openedition.org/revuehn/ 2120 ; DOI : https://doi.org/10.4000/revuehn.2120

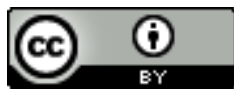

Les contenus de la revue Humanités numériques sont mis à disposition selon les termes de la Licence Creative Commons Attribution 4.0 International. 


\section{humanités \\ numériques}

3 | 2021

Humanités numériques spatialisées

\section{Éditorial. Le troisième vient, c'est encore une première... \\ Editorial. The Third Is upon Us, It's Yet Another First...}

Entrées d'index

MOTS-CLÉS : humanités numériques

KEYWORDS : digital humanities

\section{Un premier numéro thématique}

Ce troisième numéro de la revue Humanités numériques ouvre l'alternance que nous souhaitons entre recueils d'articles variés (numéros 1 et 2 , puis numéro 4 à venir, pour lequel s'achève la phase d'évaluation) et numéros consacrés à un domaine particulier. Ces derniers font l'objet de l'appel à contribution habituel et suivent la même chaîne d'évaluation et de préparation éditoriale, mais sont dirigés par des éditeurs invités. Le comité de direction de la revue tient à remercier les collègues qui ont coordonné ce numéro sur le rôle des outils et méthodes numériques dans la réflexion sur l'espace, qu'il soit géographique et objectivé ou social et vécu. Suivant le modèle qui nous semble le plus profitable, l'introduction est un article de synthèse produit par les éditeurs, qui allie des indications historiques, théoriques ou méthodologiques à la présentation de la constitution et de la structure du numéro. Nous espérons que cet état de l'art suggestif et les textes publiés seront utiles aux spécialistes comme aux non-spécialistes.

Le prochain numéro thématique, intitulé « Enseigner et apprendre les humanités numériques ", sera publié en 2022. Ce numéro 5 a d'ores et déjà trouvé ses auteurs et autrices. En attendant d'en lire les articles, vous pouvez retrouver ici l'argumentaire préparé par ses éditeurs invités, avec lesquels nous nous réjouissons de travailler. 


\section{Un premier compte rendu}

Dans cette livraison, nous inaugurons également une rubrique de comptes rendus. Y seront proposés des éclairages sur des publications francophones, le plus souvent récentes. Que vous soyez auteur ou autrice de l'ouvrage, en contact ou non avec la maison d'édition, en mesure ou non de transmettre à la revue une copie imprimée ou numérique, vos recommandations seront accueillies avec intérêt.

Il nous reste à vous souhaiter une excellente lecture numérique, en quelque point de l'espace des humanités que vous vous trouviez actuellement!

Le comité de direction : Aurélien Berra, Emmanuel Château-Dutier, Sébastien Poublanc, Émilien Ruiz, Nicolas Thély

\section{Droits d'auteur}

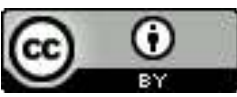

Les contenus de la revue Humanités numériques sont mis à disposition selon les termes de la Licence Creative Commons Attribution 4.0 International. 